\title{
Aerodynamic Design of a Forebody-integrated Bump Inlet With A S-Shape Diffuser
}

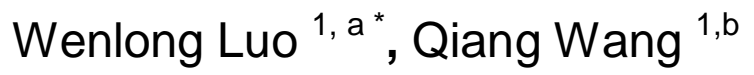 \\ ${ }^{1}$ Beihang University, Xueyuan Rd \#37, Haidian Dist, Beijing, China \\ aluo_wenlong@foxmail.com, b qwang518@buaa.edu.cn
}

Keywords: Bump Inlet, S-Shape Diffuser, Airframe-inlet Integrity Design

Abstract. In this paper the whole design method of an integrated bump inlet with an S-shape diffuser at its design Mach number 1.7 is presented. The model of the intake system with airframe is established based on the aircraft's flight task requirements. Then CFD analysis result indicates the low-momentum boundary layer fluid could be pushed away from the model centerline to the sides of the bump. The total pressure recovery reach 0.91 at the outlet of the intake. If the S-shape Diffuser is carefully designed by the function of centerline distribution, area distribution and area shape, it would prevent the airflow separation behind the normal shock wave in throat effectively and achieve better performance of the inlet.

\section{Introduction}

The bump inlet, also referred to Diverterless Supersonic Inlet, has been one of the novel intake systems for the modern supersonic aircraft. Unlike the most conventional inlet to prevent the low-momentum boundary layer flow by a diverter near the fuselage or wing, the bump inlet separate the boundary layer by the high pressure zone generated by a 3-demensional bump before the inlet. The bump inlet was firstly investigated by NASA in the 1950s. Simon et al[1] studied an external bump inlet in a direct comparison with a traditional two-dimensional compression ramp. It was determined that the bump inlet outperformed the ramp inlet over a range of Mach numbers from 1.5 to 2, with both surfaces employing boundary layer bleed. The DSI traces its roots to work done by Lockheed Martin engineers in the early 1990s as part of an independent research and development project called the Advanced Propulsion Integration project.[2]The overall inlet design moved from concept to reality when it was installed and flown on a Block 30 F-16 in a highly successful demonstration program. Presently there are 2 tactical aircraft that have successfully integrated the DSI inlet, one is F-35 Joint Strike Fighter, another is China-Pakistan co-developed JF-17 fighter. The wind tunnel test of JF-17 is conducted and analyzed by Y.K.Yang[3]. Both the test of F-16 and the JF-17 prove that the better performance in boundary separation and total pressure recovery. An US patent applications was filed in 1998, in which the method of designing a bump is introduced[4]. A study entitled "Subsonic Diffusers For Highly Survivable Aircraft" indicate that compact diffusers can be made to yield acceptable performance by proper duct shaping method. Nevertheless, a systematic method of the bump inlet with a S-shape subsonic diffuser based on the flight task of an aircraft is not presented in current study.

The present paper is based on proterotype of the JF-17 fighter. In this study the focus is on the process of design from the wave shock design of the bump to the shaping of the subsonic diffuser. Then aerodynamic performance is calculated after the model is designed. Basic flow and performance characteristic of the inlet system is evaluated at zero angle-of-attack(AOA) and zero side-slip angle. 


\section{Design Procedure}

The JF-17 is a single engine fighter and its intake system is side-mounted embodiment. Two ducts on each side merge into one and connect the entry of the engine by a straight isolator. The max flight mach number is 1.7 and the throat mach number is around 0.7. The upstream flow compress and slow down after passing a conical wave shock and a normal shock. Therefore, it is available to design the two wave shocks. Essentially, the bump inlet is still a 2 wave shock supersonic inlet. However, unlike the conventional inlet, the oblique shock is replaced by an conical shock as Fig.2. According to the shock equation:

$$
\begin{aligned}
& \rho_{1} V_{1}=\rho_{2} V_{2} . \\
& p_{1}-p_{2}=\rho_{2} V_{2}^{2}-\rho_{1} V_{1}^{2} . \\
& c_{p} T_{1}+0.5 V_{1}^{2}=c_{p} T_{2}+0.5 V_{2}^{2} .
\end{aligned}
$$

The aerodynamic coefficients before the normal shock could be calculated: the mach number is $1.3 \sim 1.4$, and the total pressure recovery is nearly 0.952 . It also means these are the aerodynamic coefficients of the airflow behind the conical shock. The conical shock generated by the bump surface could be equivalent to be generated by a cone as shown in Fig.2.And the aerodynamic coefficients behind conical shock could be calculated numerically by Taylor-Maccoll method. In the present study, a program in Matlab R2014a is coded and use to solve the flow of the conical shock and generate 20 streamline to form the bump model in Siemens NX 8.5. Given the upstream mach number 1.7 and the after-shock mach number 1.3 1.4, the semi-vertex angle is approximately 20 degrees and total pressure recovery of the shock is 0.996 .

For the engine need to obtain enough airflow in its max work, the capture area and the throat area need to be evaluated according to the corrected mass flow: $\mathrm{A}_{\mathrm{c}}=\frac{K G_{r e d} \sigma}{242 q\left(\lambda_{\infty}\right) \omega_{c r}}$.

And the throat area is

$$
A_{t h} \geq \frac{\varphi_{\max } q\left(\lambda_{\infty}\right) A_{c}}{0.98 \sigma_{s}}
$$




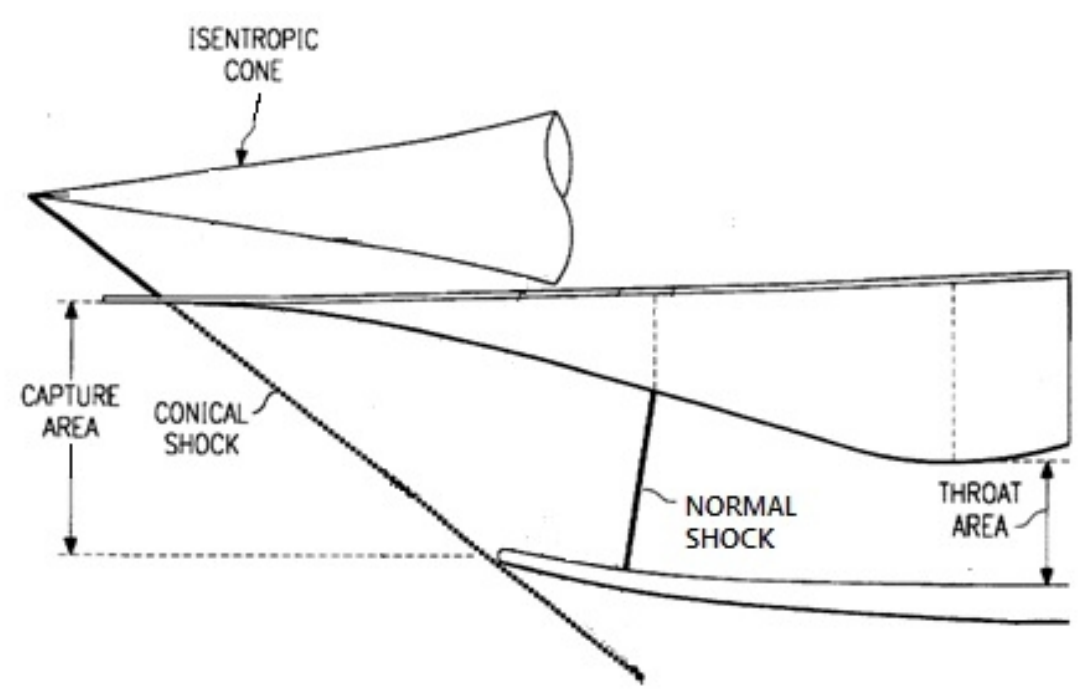

Fig. 1 The shock wave generated in an bump inlet

Unlike the conventional straight lip, the lips of the JF-17 is forward swept, which could let the boundary layer flow be push away behind the lip and bump.

The subsonic diffuser of the inlet is S-shape and the centerline of inlet entry and engine entry have a distance of $1.3 \mathrm{~m}$. A combination of a slow turning with a high diffusion rate at the entrance, which proved having the best performance[5], is adopted to design the diffuser. And their mathematical representation are: $\quad y=\Delta y\left[-3\left(\frac{x}{L}\right)^{4}+4\left(\frac{x}{L}\right)^{3}\right]$.

where $\mathrm{y}$ is the offset distance.

$$
\frac{A}{A_{1}}=\left(\frac{A_{2}}{A_{1}}-1\right)\left[3\left(\frac{x}{L}\right)^{4}-8\left(\frac{x}{L}\right)^{3}+6\left(\frac{x}{L}\right)^{2}\right]+1 \text {. }
$$

where $A_{1}$ and $A_{2}$ are areas of the diffuser entrance and exit, respectively. The A2/A1 are the Area Ratio. All the diffuser design parameter are illustrated in Fig.2.

The change regulation from the entrance to exit adopt a even method to achieve. After finishing the diffuser's design, the whole bump inlet system is modeled out as Fig.2 shows.

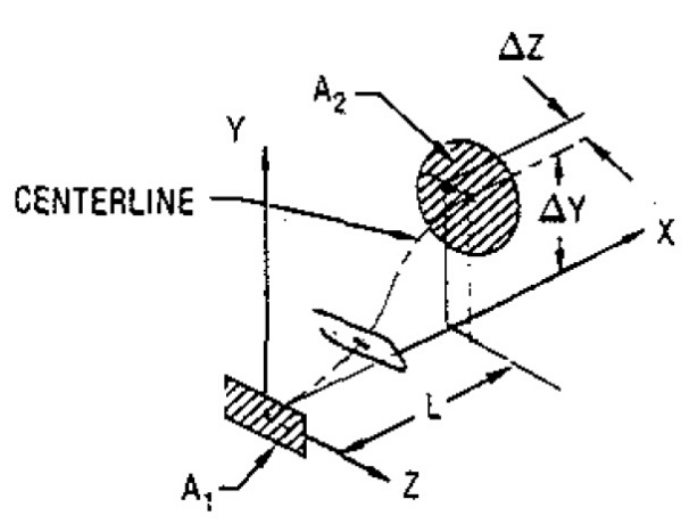

Fig.2 Diffuser Design Parameter

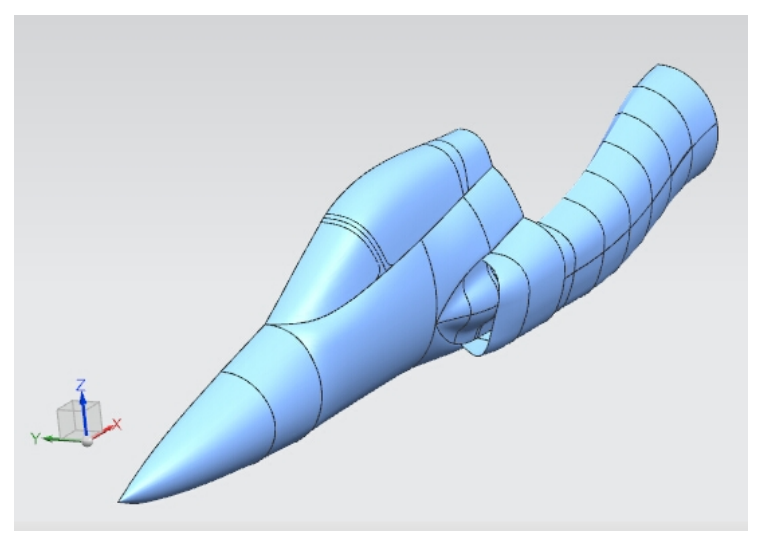

Fig.3 The Intake System Model 


\section{Computational Setup}

For the present 3D CFD analysis, left half of forward fuselage and intake are modeled with a plane of symmetry. The intake exit is modeled about one intake-diameter away from the engine face so as to avoid the effect of applied boundary conditions on flow characteristics at engine face. In addition to the forebody and intake, the complete flow domain is meshed including the free stream far field and the symmetry plane. The intake duct is meshed with structured grid and the outside free stream is meshed with unstructured grid in Pointwise v17, as show in Fig.3in the present study the total cell count is nearly 1.32 million points and the near wall distance is $0.07 \mathrm{~mm}$.

For the boundary condition, no-slip condition was enforced at the surfaces/walls of fuselage and intake. Pressure far field conditions were used at far field and fuselage base corresponding to the desired free stream mach number and angle-of-attack. Pressure outlet condition is applied at intake duct outlet to control the mass flow rate. Symmetry boundary condition was specified on the symmetry planes of the fuselage external domain.

For the present study, the two-equation SST k- $\omega$ turbulence model is used due to its demonstrated feasibility in the aerospace application. The use of a k- $\omega$ formulation in the inner parts of the boundary layer makes the model directly usable all the way down to the wall through the viscous sub-layer. Meanwhile, the SST formulation also switches to a k- $\varepsilon$ behavior in the free-stream and thereby avoids the common k- $\omega$ problem that the model is too sensitive to the inlet free-stream turbulence properties. It has good behavior in adverse pressure gradients and separating

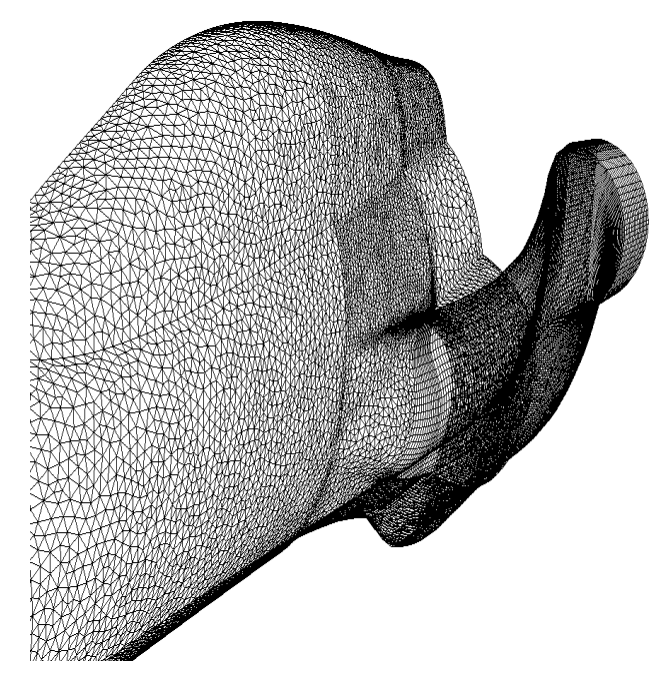

Fig.4 Mesh Of The Inlet System

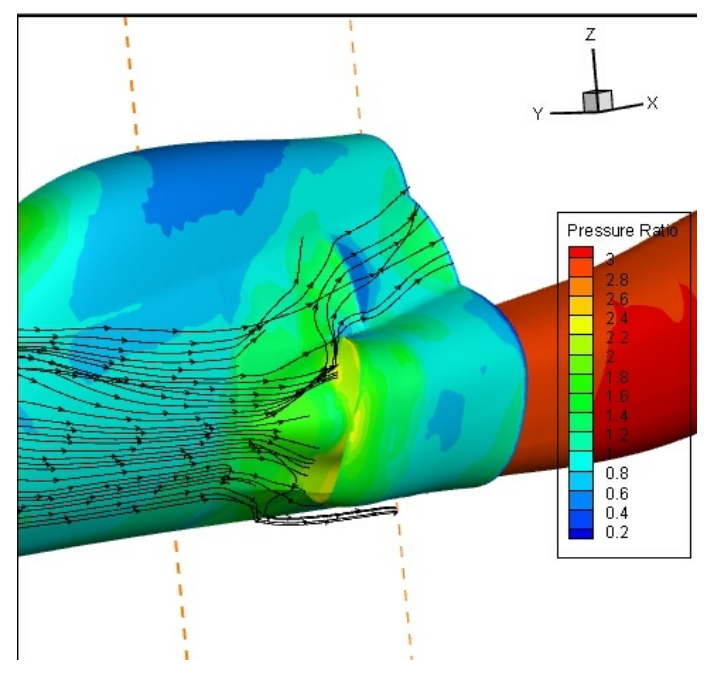

Fig.5 Pressure Contour And Streamline flow.

\section{RESULT AND DISCUSSION}

As the Fig 5 shows, at the mach number 1.7, a high pressure zone occurred in front of the entrance, where the state pressure is nearly 1.6 times than the pressure in far field . Hence, it indicates that the a well-designed 3-D bump blow the upstream boundary layer away from the inlet from behind the normal shock effectively. The same result could also be proved with the help of the flow traces figure. The upstream boundary layer diversion due the bump compression surface is evident.

The pressure distribution of the inlet and diffuser is presented in Fig.5. The flow pass the conical shock in front of the bump surface and the pressure arise nearly 1.6 time the upstream. Then flow 
pass the normal shock in front of the throat and pressure arises 2 time. In the subsonic diffuser the static pressure arise gradually, and reach nearly 3 times of upstream. The same effect of the conical shock and normal shock could be observed in the mach number contour shown in the Fig.6. After passing the conical shock, the mach number of the stream decreases from 1.7 to 1.35. After passing the normal shock, the mach number decrease to 0.7 . Then, the flow in the subsonic diffuser slow as the section expand, finally reach 0.45 at the exit of the subsonic diffuser. The contour shows that the inlet and diffuser are designed effectively to decelerate the upstream flow for the engine.

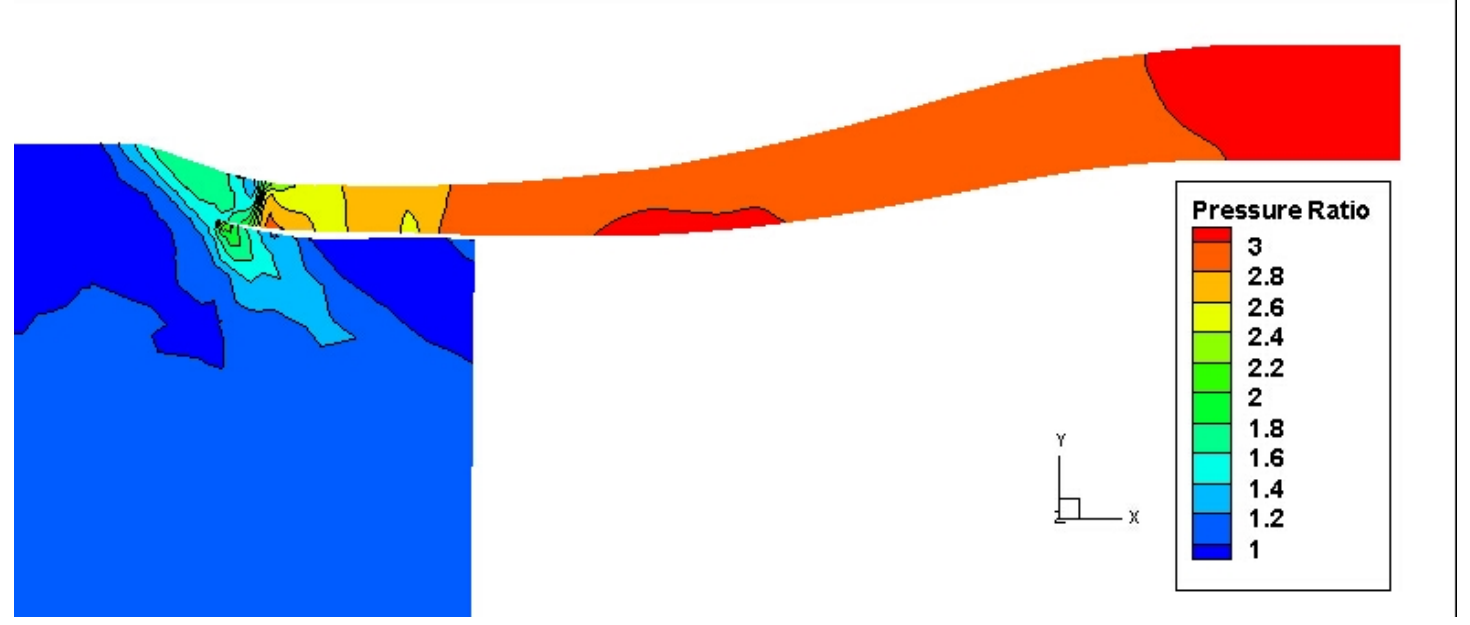

Fig.6 Static Pressure Distribution

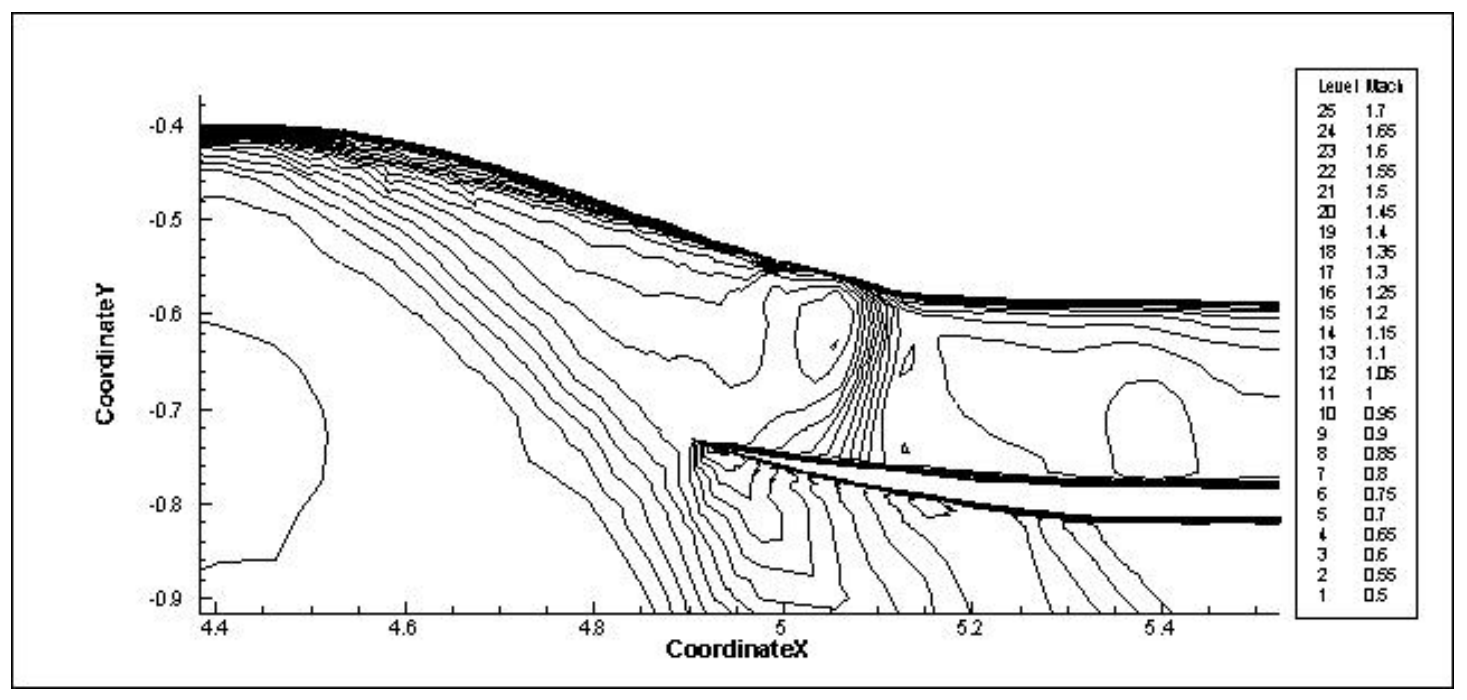

Fig.7 Mach Number Distribution

Fig. 8 shows the supersonic intake total pressure recovery at $\mathrm{Ma}=1.7$, for a range of intake mass flow ratios. At high supersonic speeds, the total recovery characteristics at the exit of the inlet is dependent on the intake duct flow but also ,to a greater extent, on the intake shock wave structure. The conventional supersonic behavior for a external compression fixed intake is that as the mass flow ratio increases from a sub-critical level, the total pressure ratio recovery also increases due to inlet shock waves adopting a structure progressively closer to the design point structure. Beyond the design point mass flow ratio(intake super critical operation), the inlet shock waves are generally 
ingested into the diffuser with rapidly deteriorating total pressure recovery. This behavior is shown as the "Conventional Inlet" line in Fig.8

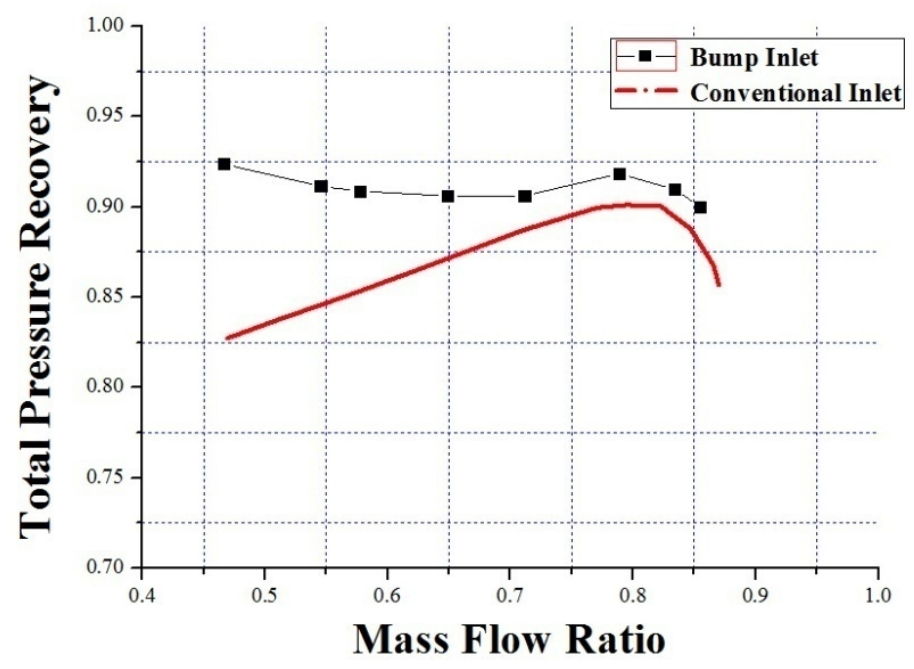

Fig.8 Total pressure recovery of the inlet model

\section{Conclusion}

In this paper the flow and performance characteristics of a forebody-integrated bump inlet are designed and analyzed. The flow details presented here are in greater detail than that can be extracted from wind tunnel test and, therefore, can be used for further design refinement. The operating mechanism of a DSI in getting rid of the upstream boundary layer at subsonic and supersonic conditions has been demonstrated and its basic pressure recovery characteristics have been quantified for both subsonic and supersonic flight conditions.

\section{Reference}

[1] Simon ,P C.; Brown, D W.; Huff, R. G. "Performance of external-compression bump inlet at Mach numbers of 1.5 and 2.0" NACA Report NACA-RM-E56L19 (1957).

[2] Hehs E. JSF diverterless supersonic inlet[ J]. Code One Magazine, 2000, 15( 3) .

[3] Yang, Y. "Design of Bump Inlet of Thunder / JF-17 Aircraft" Journal of Nanjing University of Aeronautics \& Astronautics, Vol. 39, No. 4, August 2007, pp. 449-452.

[4] Hamstra, J. W, McCallum, B.N, Sylvester, T.G, Denner, B.W, and Moorehouse, J. A., "Transition Shoulder System andMethod for Diverting Boundary Layer Air," U.S. Patent $5,749,542,1998$.

[5] Lee C C, Boedicker C. Subsonic diffuser design and performancefor advanced fighter aircraft[J] AIAA-85-3073, 1985. 